\title{
Generation of Polyclonal CMV-specific T Cells for the Adoptive Immunotherapy of Glioblastoma
}

\author{
Alexia Ghazi ${ }^{1,2,3}$, Aidin Ashoori ${ }^{1,2,3}$, Patrick Hanley ${ }^{1,3}$, Vita S Salsman ${ }^{1,2,3}$, Donald R \\ Shaffer ${ }^{1,3}$, Yvonne Kew ${ }^{6}$, Suzanne Z Powell ${ }^{7}$, Robert Grossman ${ }^{6}$, Zakaria Grada ${ }^{1,2,3}$, \\ Michael E Scheurer ${ }^{1,2,3}$, Meenakshi Hegde ${ }^{1,2,3}$, Ann M Leen ${ }^{1,2,3}$, Catherine M Bollard ${ }^{1,2,3,4,5}$, \\ Cliona M Rooney ${ }^{1,3,4}$, Helen E Heslop ${ }^{1,2,3,5}$, Stephen Gottschalk ${ }^{1,2,3,4}$, and Nabil Ahmed 1,2,3' \\ ${ }^{1}$ Center for Cell and Gene Therapy, Baylor College of Medicine, The Methodist Hospital and \\ Texas Children's Hospital, Houston, Texas 77030, USA \\ ${ }^{2}$ Texas Children's Cancer Center, Baylor College of Medicine, Houston, Texas 77030, USA \\ ${ }^{3}$ Department of Pediatrics, Baylor College of Medicine, Houston, Texas 77030, USA \\ ${ }^{4}$ Department of Pathology and Immunology, Baylor College of Medicine, Houston, Texas 77030, \\ USA \\ ${ }^{5}$ Department of Medicine, Baylor College of Medicine, Houston, Texas 77030, USA \\ ${ }^{6}$ Department of Neurosurgery, The Methodist Hospital, Houston, Texas, USA \\ ${ }^{7}$ Department of Pathology, The Methodist Hospital, Houston, Texas, USA
}

\section{Abstract}

Glioblastoma (GBM) is the most common primary brain cancer in adults and is virtually incurable. Recent studies have shown that CMV is present in the majority of GBMs. To evaluate if the CMV antigens pp65 and IE1, which are expressed in GBMs, can be targeted with CMVspecific T cells, we measured the frequency of T cells targeting pp65 and IE1 in the peripheral blood of a cohort of 11 sequentially-diagnosed CMV-seropositive GBM patients, and evaluated whether it was feasible to expand autologous CMV-specific T cells for future clinical studies. All 11 CMV-seropositive GBM patients had T cells specific for pp65 and IE1 in their peripheral blood assessed by IFN $\gamma$ ELIspot assay. However, the precursor frequency of pp65-specific T cells was decreased in comparison to healthy donors $(p=0.001)$. We successfully reactivated and expanded CMV-specific T cells from 6 out of 6 GBM patients using antigen presenting cells transduced with an adenoviral vector encoding pp65 and IE1. CMV-specific T-cell lines contained CD4-positive as well as CD8-positive T cells, recognized pp65- and IE1-positive targets and killed CMV-infected autologous GBM cells. Infusion of such CMV-specific T-cell lines may extend the benefits of Tcell therapy to patients with CMV-positive GBMs.

\section{Keywords \\ Glioblastoma; GBM; CMV; T cells; immunotherapy; pp65; IE1}

Correspondence should be addressed to: Nabil Ahmed, MD, MPH, Phone: 832-824-4611, Fax: 832-825-4732, nahmed@bcm.edu, Center for Cell and Gene Therapy, Baylor College of Medicine, 1102 Bates Street; MC 3-3320, Houston, TX 77030.

Publisher's Disclaimer: This is a PDF file of an unedited manuscript that has been accepted for publication. As a service to our customers we are providing this early version of the manuscript. The manuscript will undergo copyediting, typesetting, and review of the resulting proof before it is published in its final citable form. Please note that during the production process errors may be discovered which could affect the content, and all legal disclaimers that apply to the journal pertain.

Financial Disclosure: All authors have declared there are no financial conflicts of interest in regards to this work 


\section{Introduction}

Glioblastoma (GBM) is the commonest primary brain cancer and is virtually incurable. $(1 ; 2)$ Of an estimated 22,020 brain tumors diagnosed in the United States in 2010, there were approximately 9,000 GBMs. The current standard of care for newly diagnosed GBM is gross surgical resection, followed by adjuvant radical radiotherapy and temozolomide.(3) For newly diagnosed patients, the median survival is approximately one year from the time of diagnosis, and more than $85 \%$ of patients are expected to die within two years, with fiveyear survival rates of less than 4\%.(1;4) Given this dismal outcome, novel biologicallybased therapies are needed to improve survival for GBM patients. Targeted immunotherapy has this potential.

CMV is a ubiquitous $\beta$-herpesvirus that during primary infection can produce a minor mononucleosis-like syndrome in the immuno-competent host followed by life-long latency. Several recent reports have demonstrated the presence of CMV in 80-100\% of GBMs as judged by the presence of the CMV proteins pp65 and IE1 using immunohistochemistry (IHC). (5-8) In addition, CMV DNA as well as viral particles were detected in pp65- and IE1-positive cells. ${ }^{(5 ; 6)}$ It is possible that CMV promotes the malignant phenotype of GBM cells by enhancing cell invasiveness and activating telomerase.(9-11)

The clinical experience with immunotherapy targeting CMV in GBM patients is limited. In the only published clinical study in abstract form, newly-diagnosed GBM patients were vaccinated with pp65-RNA-transfected DCs after completion of front-line therapy. The vaccine was well tolerated and patients had a prolonged progression-free survival in comparison to historic controls. (12) Adoptive T-cell therapy provides and alternative means to target virus-associated diseases and cancers. (13-16) Adoptive transfer of donor derived T cells specific for adenovirus (Ad), cytomegalovirus (CMV), and Epstein Barr virus (EBV) post hematopoietic stem cell transplantation (HSCT) is safe, reconstitutes anti-viral immunity, and has antiviral effects. (17) In addition, EBV-specific T cells have antitumor activity against EBV-positive malignancies post transplant as well as outside the transplant setting. (15-20)

To evaluate if the adoptive transfer of CMV-specific T cells in GBM patients is feasible, we determined if autologous CMV-specific T cells could be generated from patients using our Good Manufacturing Practice (GMP)-compliant method and characterized their function.

\section{Materials and Methods}

\section{Study subjects}

Newly-diagnosed GBM patients were consented on a Methodist Hospital and Baylor College of Medicine IRB-approved protocol. The study cohort consisted of 20 sequentiallydiagnosed patients ( 8 female and 12 male) with a median age of 50.1 years (range 37-72 years). CMV IgG titres were performed on 20 serum samples of this cohort. Of these, IHC and ISH were performed on 11 paraffin-embedded slide sets from 11 CMV seropositive patients. CTL lines were initiated on 6 of these patients using peripheral blood mononuclear cells.

\section{Immunohistochemistry}

Formalin-fixed, paraffin embedded sections ( $6 \mu \mathrm{m})$ of primary human GBM were used. (21) Known CMV-infected lung samples were used as a positive tissue control for all experiments. All slides were deparaffinized by heating slides in a xylene bath at $50^{\circ} \mathrm{C}$ for 1 hour and 10 minutes, followed by a 30-minute incubation period at room temperature. The 
slides were then washed additionally in xylene and serially diluted in ethanol baths $(100,95$, 70 and 50\%), post-fixed in neutral buffered formalin and treated for pepsin digestion (BioGenex, San Ramon, CA, USA). Freshly prepared $30 \% \mathrm{H}_{2} \mathrm{O}_{2}$ was used to block endogenous peroxidase before performing antigen retrieval using CitraPlus antigen retrieval solution (BioGenex) for 2.5 hours at $50^{\circ} \mathrm{C}$. Avidin, biotin (BioGenex) and $\mathrm{Fc}$ receptor (Innovex Biosciences, Richmond, CA, USA) blocking reagents were applied to the sections prior to a $4^{\circ} \mathrm{C}$ overnight incubation with anti-IE172 (1:100; Chemicon, Temecula, CA, USA) and anti-pp65 (1:40; Leica Microsystems Inc., Bannockburn, IL, USA) primary antibodies. Positive control sections were treated with anti-actin monoclonal antibody (1:35; BioGenex), while negative control sections were similarly incubated with no primary antibody. The sections were then developed using a biotinylated anti-mouse secondary antibody (1:16.5; BioGenex), peroxidase-labeled streptavidin (Biogenex) and 3,3'diaminobenzidine (Innovex Biosciences) as a chromogen. All slides were counterstained in Harris hematoxylin, dehydrated and coverslipped.

\section{In situ hybridization}

All paraffin-embedded sections $(6 \mu \mathrm{m})$ were deparaffinized and post-fixed in neutral buffered formalin, similar to the sections for IHC. (21) Pepsin digestion, endogenous peroxidase block and antigen retrieval were also performed for the ISH sections as described above. A human CMV DNA probe cocktail consisting of 12 oligonucleotides end-labeled with five fluorescein-linker molecules (BioGenex) was used to detect human CMV DNA in the sections. A probe mix containing two 20-mer probes against the endogenous Alu DNA sequence (BioGenex) was used as a positive control. A probe against the reverse complementary sequence of black beetle virus RNA2 sequence (BioGenex) was used as a nonspecific negative control. Enzyme digestion was performed at $37^{\circ} \mathrm{C}$ for $4 \mathrm{~min}$, and nucleic acid denaturation was performed at $90^{\circ} \mathrm{C}$ for $20 \mathrm{~min}$ on a slide moat (Boekel Scientific, Feasterville, PA, USA). The slides were then hybridized with each probe at $37^{\circ} \mathrm{C}$ in a humidified chamber overnight. This probe cocktail was optimized by the manufacturer for hybridization at $37^{\circ} \mathrm{C}$ overnight in a 50\% formamide solution. Low-concentration probes in 50\% formamide with overnight incubation are known to ensure the highest specificity and lowest background for ISH reactions. (21) In addition, a triple stringency wash (rinse with $2 \times$ phosphate-buffered saline followed by $0.0025 \times$ standard saline citrate at $40^{\circ} \mathrm{C}$ for $20 \mathrm{~min}$ and Tris-buffered saline with Triton $\mathrm{X}$ ) was performed to remove any nonspecific hybrid pairs. Probes were detected using a sensitive detection system consisting of a mouse antifluorescein antibody (BioGenex), biotinylated anti-mouse $\mathrm{F}(\mathrm{ab}) 2$ fragments (BioGenex) and an alkaline phosphatase-streptavidin label using the chromogen nitro-blue tetrazolium/5bromo-4-chloro-3-indolyl phosphate (NBT/BCIP) as a substrate (BioGenex). The slides were then counterstained with Nuclear Fast-Red (Sigma).

\section{Blood donors, primary tumor cells and cell lines}

Blood samples and primary tumor cells were obtained from subjects with GBM on a protocol approved by the Institutional Review Board of Baylor College of Medicine and The Methodist Hospital. The GBM cell line U373 and the breast cancer cell line (MDAMB-468) were purchased from the American Type Culture Collection (ATCC; Manassas, VA). All cell lines were grown in DMEM (Invitrogen, Carlsbad, CA) with 10\% fetal calf serum (FCS; HyClone, Logan, UT), supplemented with $2 \mathrm{mM}$ GlutaMAX-I, $1.5 \mathrm{~g} / \mathrm{L}$ sodium bicarbonate, $0.1 \mathrm{mMol} / \mathrm{L}$ nonessential amino acids, and $1.0 \mathrm{mMol} / \mathrm{L}$ sodium pyruvate (all media supplements from Invitrogen). T cells were maintained in RPMI 1640 with 10\% FCS containing $2 \mathrm{mMol} / \mathrm{L}$ GlutaMAX-I.

Tumor tissues from patients with GBM undergoing surgical resection were processed aseptically, and primary cell cultures were initiated using DMEM high glucose medium 
(Invitrogen), supplemented with 15\% heat inactivated FCS, 2 mM GlutaMAX-I, 1\% InsulinTransferrin-Selenium-X supplement, and 1\% Penicillin-Streptomycin mixture (all media supplements from Invitrogen). Cells were used within 7 days of plating or established as primary cell lines. (22)

\section{Generation of CMV-specific T cells from GBM patients}

Frozen PBMCs were thawed, washed, and resuspended at $1 \times 10^{6}$ cells $/ \mathrm{mL}$ in a 24 -well plate in X-Vivo 15 media (Lonza Bioscience, Basel) supplemented with GlutaMAX-I (Invitrogen, Carlsbad, USA) overnight to activate monocytes. $(17 ; 23)$ The next day adherent and nonadherent cells were harvested, and transduced with an adenoviral vector encoding IE1 and pp65 separated by an internal ribosomal entry site (Ad5f35-IE-I-pp65) at a multiplicity of infection (MOI) of 2500 viral particles (vp)/cell for 90 minutes at $37^{\circ} \mathrm{C}$. (24) Cells were then resuspended in 45\% RPMI (Hyclone) and 45\% CLICKS (Irvine Scientific) with 10\% human serum plus GlutaMAX-I (CTL medium) at $2 \times 10^{6}$ cells/well in a 24 -well plate. Cultures were restimulated on day 10 and then weekly at a responder-to-stimulator ratio of 4:1 with irradiated, autologous lymphoblastoid cell lines (LCL), which were transduced with Ad5f35-IE1-I-pp65. IL-2 (50-100 U/mL; Proleukin; Chiron) was added 3 days after the second stimulation and then twice weekly.

\section{Flowcytometry}

T-cell lines were analyzed with monoclonal antibodies to CD19, CD4, CD8, CD56/16, TCR $\alpha \beta, T C R \gamma \delta, C D 3 / 28$, CD62L, CD4 (Becton Dickinson). Samples were acquired on a FACScan flow cytometer (Becton Dickinson). The data were analyzed with the use of CellQuest software (Becton Dickinson). To detect pp65- specific T cells in the T-cell lines, we used pentamers specific for the MHC class I-restricted pp65 epitopes HLA-A1 YSEHPTFTSQY, HLA-A2 NLVPMVATV, HLA-A24 QYDPVAALF, and HLA-B7 TPRVTGGGAM (Proimmune, Sarasota, FL). Pentamer staining of T cells $\left(5 \times 10^{5}\right)$ was done as previously described. (24) For each sample, 100,000 cells were analyzed using CellQuest software.

Intracellular cytokine staining was used to determine the frequency of CMV-specific $\mathrm{T}$ cells. Briefly, T cells were incubated for one hour with PepMixes encoding the CMV antigens pp65 or IE1 (JPT Peptide Technologies, Berlin, Germany) in the presence of costimulatory antibodies CD28 and CD49d (BD Bioscience) before adding Monesin and Brefeldin A (BD Bioscience) to block cytokine secretion. ${ }^{20,22}$ After an additional $4 \mathrm{~h}$ incubation, $\mathrm{T}$ cells were stained with CD3 and CD8, and subsequently permeabilized using Cytofix/Cytoperm (BD Bioscience). Intracellular cytokines were detected using monoclonal antibodies to IFN $\gamma$, and TNF $\alpha$.

\section{Enzyme-linked immunospot assay}

Enzyme-linked immunospot (ELIspot) analysis was used to determine the frequency and function of T cells secreting IFN $\gamma$ in response to PepMixes encoding CMV antigens (pp65, IE1), Ad antigen (hexon), and EBV antigens (BZLF1, EBNA3c). All PepMixes used contained 15 amino-acid peptides covering the entire length of the corresponding protein with an 11 amino-acid overlap (JPT Peptide Technologies, Berlin, Germany). ELISPOT assays were performed on the CTL lines. (17) PBMCs stimulated with staphylococcal enterotoxin B ( $1 \mu \mathrm{g} / \mathrm{mL}$; Sigma-Aldrich) served as positive controls. Spot-forming cells were enumerated by Zellnet Consulting (Fort Lee, NJ) and compared with input cell numbers to obtain the frequency of virus-reactive $\mathrm{T}$ cells. 


\section{Cytotoxicity assay}

T-cell lines were tested for specific cytotoxicity against autologous LCLs and autologous tumor cells pulsed with IE1 and pp65 PepMix (JPT), or Adv-hexon PepMix. (24) As control target cells we used unpulsed PHA blasts, PHA blasts pulsed with irrelevant peptides, and HLA class I-mismatched LCLs. ${ }^{51} \mathrm{Cr}$-labeled target cells were mixed with effector cells at doubling dilutions to produce the effector-to-target $(\mathrm{E} / \mathrm{T})$ ratios specified. Target cells incubated in complete medium or 5\% Triton X-100 (Sigma-Aldrich) were used to determine spontaneous and maximal ${ }^{51} \mathrm{Cr}$ release, respectively. After 4 hours (LCLs and PHA blasts) or 6 hours (fibroblasts), supernatants were collected, and radioactivity was measured on a gamma counter. The mean percentage of specific lysis of triplicate wells was calculated as $100 \times($ experimental release - spontaneous release $) /($ maximal release - spontaneous release).

Using the same methodology as above, CTLs were also tested against the following GBM targets: autologous GBM lines pulsed with CMVpp65 (8) and CMV IE1 PepMixes, and autologous GBM cells and HLA-mismatched U373 cells infected overnight with CMV strain VR1814 (gift from Dr. Giuseppe Gerna and Daniele Lilleri from Pavia, Italy). Supernatants were collected, and radioactivity was measured on a gamma counter as described above. The mean percentage of specific lysis of triplicate wells was determined using the same calculation described above.

\section{Statistical analysis}

The Student's t test was used to test for significance in each set of values, assuming equal variance. Mean values plus or minus SDs are given unless otherwise stated.

\section{Results \\ Detection of CMV in primary Glioblastoma}

To confirm the presence of CMV in primary GBM samples we performed IHC for pp65 and IE1, and ISH on paraffin embedded samples from 11 serially-diagnosed, CMV-seropositive GBM patients. Ten (91\%) of 11 GBMs screened were positive for either pp65 $(5 / 11 ; 45 \%)$ or IE1 $(10 / 11 ; 91 \%)$. The pattern of pp65 and IE1 expression was similar to previous reports with pp65 expression being primarily nuclear and IE1 diffusely cytoplasmic (Figure 1A and figure, Supplemental Digital Content 1). $(5 ; 6 ; 8)$ For 9 samples we had sufficient material to perform ISH using a human CMV DNA probe cocktail consisting of 12 oligonucleotides. Eight (89\%) of 9 GBMs were positive for the CMV genome (Figure 1B and figure Supplemental Digital Content 2). These results confirm our previously published findings and those from others that CMV proteins (early and late) as well as the CMV genome are detectable in the majority of primary GBM samples. $(8 ; 21 ; 25)$

\section{CMV-seropositive GBM patients have IFNy secreting pp65- and IE1-specific T cells in their peripheral blood}

The cellular immune response to pp65 and IE1 in peripheral blood of GBM patients has not been studied. We therefore determined the precursor frequency of pp65- and IE1-specific T cells in the peripheral blood of our 11 GBM patients and 7 healthy controls using pp65 and IE1 Pepmixes in IFN $\gamma$ ELIspot assays. We observed a significantly lower median precursor frequency of pp65-specific T cells in GBM patients $(n=11)$ in comparison to healthy controls ( $\mathrm{n}=8$ ) (Figure 2A; median 20 (range 2-269) SFC per $2 \times 10^{5}$ PBMC vs. median 408 (range 21-710) SFC per $2 \times 10^{5}$ PBMC, respectively; $p=0.001$ ). Individual GBM patients had a lower frequency of pp65-specific T cells regardless of the presence of pp65 in their tumor tissue. In contrast to pp65, the precursor frequency of IE1-specific T cells was similar in both groups. (Figure 2A; median 26 (range 1-367) SFC per $2 \times 10^{5}$ PBMC vs. median 49 
(range 13-151) SFC per $2 \times 10^{5}$ PBMC, respectively; $p=0.49$ ). Figure $2 \mathrm{~B}$ summarizes the pp65 and IE1 IHC and CMV ISH data performed on tumor tissues and the precursor frequency of pp65- and IE1- specific T cells in the peripheral blood of the studied GBM patients at baseline. All 11 GBMs were uniformly positive for the CMV genome, IE1 and/or pp65 except for the GBM of patient number 6, which only showed focal staining for IE1. In 8 of 9 GBM patients the pp65-specific T-cell precursor frequency was below 1 standard deviation (SD) in comparison to healthy controls. In contrast only 2 had an IE1-specific Tcell precursor frequency, which was below $1 \mathrm{SD}$.

\section{Successful ex vivo expansion of pp65 and IE1-specific CTL from GBM patients}

So far we have shown that patients' GBM express pp65 and IE1 and that pp65 and IE1specific $\mathrm{T}$ cells are present, albeit at a lower frequency for pp65 than normal controls, in their peripheral blood. We next determined if it would be feasible to reactivate and expand sufficient number of pp65 and IE1-specific T cells from GBM patients using our GMPapproved method, which we had established to expand polyclonal pp65 and IE1-specific T cells from healthy donors. (24) Using this method, we successfully expanded CMV-specific T cells from 6 of 6 GBM patients. Median T-cell expansion was 123-fold (Range: 32-265; Figure 3A) within 6 weeks of culture, which was similar to the expansion of previously reported healthy controls. (24) Patients' T-cell lines contained a high percentage of TCR $\alpha \beta$, CD3-positive T cells [mean, 98.8\%; standard deviation (sd), $0.94 \%$; Figure 3B] with a CD8 [mean, 67.7\%; sd, 17.4\%] as well as a CD4 component [mean, 44\%; sd 27.7\%].

To determine if the expanded T-cell lines contained pp65- and IE1-specific T cells we performed IFN $\gamma$ ELIspot assays using pp65 and IE1 pepmixes as stimulators. All 6 CTL lines contained T cells specific for pp65 (median 292 SFC per $1 \times 10^{5}$; range 71-960) and IE1 (median 549 SFC per $1 \times 10^{5}$; range 52-697) (Figure 4A). Responses for both antigens were significantly above controls (median 32 SFC per $1 \times 10^{5}$; range $12-82 ; \mathrm{p}=0.01$ and $\mathrm{p}=0.005$; respectively). The presence of pp65-specific $\mathrm{T}$ cells was confirmed in a subset of cell lines with informative HLA-restricted pentamers (Figure 4B). These results indicate the successful expansion of pp65 and IE1-specific T cells from GBM patients' peripheral blood.

While our pp65 and IE1-specific T-cell expansion protocol preferentially activates CMVspecific $\mathrm{T}$ cells, it also activates $\mathrm{T}$ cells specific for Ad and EBV antigens. We therefore determined the frequency of T cells specific Ad antigen (hexon) and EBV antigens (BZLF1 and EBNA3c). Five out of 6 cell lines contained T cells specific for Ad and EBV antigens (hexon: median 65 SFC per $1 \times 10^{5}$; range 29-69; BZLF1: median 116 SFC per $1 \times 10^{5}$; range 116-451, EBNA3c: median 122 SFC per $1 \times 10^{5}$; range 10-193) (Figure 4A). Importantly, as for healthy donors, $\mathrm{T}$ cells directed against CMV antigens dominated the T-cell lines, indicating no selective functional impairment of CMV-specific T cells in GBM patients ex vivo. $(17 ; 24)$ In addition, 35 to $42 \%$ of CMV-specific T cells secreted both effector cytokines IFN $\gamma$ and TNF $\alpha$ upon stimulation with pp65 and IE1 pepmixes, indicating polyfunctionality (Figure 5). (26;27)

\section{GBM patients' CMV-specific T cells recognize and kill CMV-expressing autologous tumor cells}

While CMV is ubiquitously expressed in primary GBM samples, the viral genome is rapidly lost upon ex vivo culture. (28) Since we first had to establish autologous LCLs before we could generate CMV-specific T-cell lines, it was not feasible to test their cytolytic function against freshly isolated, autologous GBM cells from surgical specimens. We therefore measured direct cytotoxicity of patients GBM\#1 and GBM\#2 against autologous cultured GBM cells loaded with pp65 and IE1 pepmixes in a standard ${ }^{51} \mathrm{Cr}$-release assay (Figure 6A). Both T-cell lines had cytolytic activity against peptide-loaded autologous tumor cells. 
In contrast non-peptide loaded GBM cells were not killed. Pp65- and IE1-specific T cells also killed autologous, HLA-matched GBM cells, which were infected with CMV VR1814. In contrast CMV VR1814 infected, HLA-mismatched U373 glioma cells were not killed, indicating that these cells can recognize autologous GBM cells, which endogenously expressing pp65 and IE1, in an HLA-restricted fashion (Figure 6B).

\section{Discussion}

We confirm that the immuno-dominant CMV proteins, pp65 and IE1, as well as the CMV genome are present in the majority of primary GBM samples in a cohort of sequential newly diagnosed CMV-seropositive patients. We demonstrate that it is feasible to generate pp65and IE1-specific T-cell lines from these GBM patients under GMP conditions that are appropriate for safety and efficacy testing in a future phase I/II clinical trial. Generated CMV-specific T-cell lines were reactive to pp65- and IE1-derived pepmixes and killed autologous GBM cells infected ex vivo with CMV.

Forty-five percent of GBMs in our study were pp65 positive, which is within the range (50 $70 \%$ ) reported in the literature. ${ }^{(5-8)}$ Our finding that $91 \%$ of GBMs are IE1 positive is consistent with 3 out of 4 published studies. ${ }^{(5-8)}$ One study reported a significant lower frequency of IE1 positivity (16\%), which is most likely due to differences in the used detection methods and/or scoring systems. (8) While CMV has been unequivocally detected in GBMs, its exact role in gliomagenesis is the subject of ongoing investigations. CMV genes have been implicated in promoting the malignant phenotype of GBMs as well as the immunosuppressive microenvironment. CMV genes have been implicated in promoting the malignant phenotype of GBMs as well as the immunosuppressive microenvironment. (29)

Systemic immune dysfunction has been reported in GBM patients, including increased numbers of regulatory $\mathrm{T}$ cells and monocytes with myeloid derived suppressor cell phenotype in peripheral blood, and decreased expression of CD3 $\zeta$ in Tcells. (30-33) Here we show that the precursor frequency of pp65-specific T cells in peripheral blood of GBM patients was significantly lower in comparison to a cohort of healthy individuals. Selective loss of virus-specific T-cell function has been reported for patients with other viralassociated malignancies. For example, patients with EBV-positive Hodgkin's disease (HD), who also have evidence of systemic immune dysfunction, display a selective functional impairment of T cells specific for the EBV antigens expressed in HD (LMP1 and LMP2) with active disease, which is reversed in remission. (34) Interestingly, we only observed a decreased precursor frequency of T cells specific for pp65 and not for the other CMV protein, IE1, which is more frequently expressed in GBM. Since pp65 prevents antigen presentation of IE1, the latter may never be effectively presented to the immune system escaping anergy induction. (35;36) Alternatively, this might reflect differences in the temporal expression pattern of IE1 and pp65 during the CMV life cycle or an attempt of the virus to cordon the immune response in order to complete its life-cycle. While at present the definite reason for this pp65-specific immune dysfunction remains elusive, it represents a clear justification for our attempts to reconstitute CMV-specific immunity as a therapeutic approach to GBM.

The majority of immunotherapeutic approaches for CMV-associated diseases have focused on targeting the immunodominant pp65 protein. $(17 ; 37)$ However reports also indicate that $\mathrm{T}$ cells specific for IE1 are important for CMV-protective immune reconstitution post stem cell transplantation. (38) Since GBM cells express pp65 and/or IE1 we generated CMVspecific T cells against both antigens. To generate the broadest immune response we used APCs that were genetically modified with a recombinant adenoviral encoding full-length IE1 and pp65. Using APCs, which endogenously express antigens, should also reduce the 
risk of expanding low avidity T cells, which has been observed with peptide-loaded APCs.

We successfully activated and expanded polyclonal CMV-specific T-cell lines from 6 out of 6 GBM patients using antigen presenting cells (APCs), transduced with a recombinant Ad5f35 encoding IE1 and pp65, in the presence of IL-2. CMV-specific T-cell lines expanded to a projected median of $1 \times 10^{9}$ (range $9.3 \times 10^{8}$ to $2.6 \times 10^{9}$ ) cells after 5 weeks of T-cell initiation from a typical $90 \mathrm{~mL}$ blood draw from subjects on clinical trial. The degree of expansion was comparable to that of T cell lines from healthy donors. (24) Expanded cell lines contained functional pp65- and IE1-specific $\mathrm{T}$ cells as judged by their ability to i) secrete cytokines in response to pp65 and IE1 pepmixes and ii) kill CMV-positive target cells. T cells specific for IE1 were more frequent than for pp65 in the T-cell product (median IE1:pp65 SFC ratio: 1.9:1), which is in contrast to our previously published results for healthy donors using a similar CMV-specific T-cell expansion protocol (median IE1:pp65 SFC ratio: 1:1.8). ${ }^{20}$ This difference most likely reflects the lower precursor frequency of pp65-specific T cells in the peripheral blood of GBM patients in comparison to healthy donors.

Our T-cell generation protocol, not only expanded T cells specific for CMV, but also for EBV and Ad. As for healthy donors, T cells specific for CMV had the highest frequency in cell products from GBM patients despite the observed reversal of immuno-dominance between IE1 and pp65. These results indicate that any functional loss of CMV-specific T cells can be overcome by ex vivo culture as reported for EBV-specific T cells in patients with EBV-positive HD. (40)

We will evaluate the safety and efficacy of CMV-specific T cells for GBM patients in a recently opened Phase I/II clinical study (NCT01205334). In regards to the safety, the experience with EBV-specific $\mathrm{T}$ cells for the treatment of EBV-associated malignancies is encouraging, especially since no dose limiting toxicity was observed in patients who received EBV-specific T cells for the therapy of CNS lymphomas. (41) In addition, CMVspecific $\mathrm{T}$ cells, infused to stem cell transplant recipient with CMV reactivation, and a CMVpp65-targeted DC vaccine in GBM patients have an encouraging safety profile. $(17 ; 42 ; 43)$ While on the CMVpp65-targeted DC GBM vaccine trial a prolonged progression-free survival in comparison to historic controls was observed (43) the antitumor activity of CMV-specific T cells cannot be determined prior to the clinical study due to the lack of adequate animal models. If limited efficacy of CMV-specific T cells is observed in the clinic, ex vivo genetic modification of CMV-specific T cells would not only allow targeting of non-viral tumor antigens expressed in GBMs to circumvent immune escape, but also to render $\mathrm{T}$ cells resistant to the immunosuppressive microenvironment. (22)

In summary, we demonstrate that it is feasible to generate polyclonal CMV-specific T-cell lines from CMV-seropositive GBM patients using APCs transduced with an adenoviral vector encoding IE1 and pp65. A clinical study evaluating the safety and efficacy of CMVspecific $\mathrm{T}$ cells in GBM patients is in progress.

\section{Supplementary Material}

Refer to Web version on PubMed Central for supplementary material.

\section{Acknowledgments}

We thank Dr. Eric Yvon for the helpful discussion and advice. We would also like to thank Ms. Awateef Akrabi and Ms. Reshma Kulkarni for assistance with FACS analysis and Ms. Deborah Marquez-Do and Ms. Anne Truong for assistance with IHC and ISH staining. 
Support: The authors were supported by the Virginia and LE Simmons Family Foundation, The Clayton Foundation for Research and The American Brain Tumor Association. MES is supported by K07CA131505. HEH is supported by a Dan L Duncan Chair and P30CA125123.

\section{Reference List}

1. Buckner JC. Factors influencing survival in high-grade gliomas. Semin Oncol. 2003; 30:10-14. [PubMed: 14765378]

2. Combs SE, Heeger S, Haselmann R, et al. Treatment of primary glioblastoma multiforme with cetuximab, radiotherapy and temozolomide (GERT)--phase I/II trial: study protocol. BMC Cancer. 2006; 6:133. [PubMed: 16709245]

3. Stupp R, Mason WP, Van den Bent MJ, et al. Radiotherapy plus concomitant and adjuvant temozolomide for glioblastoma. N Engl J Med. 2005; 352:987-996. [PubMed: 15758009]

4. DeAngelis LM. Brain tumors. N Engl J Med. 2001; 344:114-123. [PubMed: 11150363]

5. Scheurer ME, Bondy ML, Aldape KD, et al. Detection of human cytomegalovirus in different histological types of gliomas. Acta Neuropathol. 2008; 116:79-86. [PubMed: 18351367]

6. Mitchell DA, Xie W, Schmittling R, et al. Sensitive detection of human cytomegalovirus in tumors and peripheral blood of patients diagnosed with glioblastoma. Neuro Oncol. 2008; 10:10-18. [PubMed: 17951512]

7. Cobbs CS, Harkins L, Samanta M, et al. Human cytomegalovirus infection and expression in human malignant glioma. Cancer Res. 2002; 62:3347-3350. [PubMed: 12067971]

8. Lucas KG, Bao L, Bruggeman R, et al. The detection of CMV pp65 and IE1 in glioblastoma multiforme. J Neurooncol. 2011; 103(2):231-238. [PubMed: 20820869]

9. Straat K, Liu C, Rahbar A, et al. Activation of telomerase by human cytomegalovirus. J Natl Cancer Inst. 2009; 101:488-497. [PubMed: 19318640]

10. Cobbs CS. Evolving evidence implicates cytomegalovirus as a promoter of malignant glioma pathogenesis. Herpesviridae. 2011; 2:10. [PubMed: 22030012]

11. Soroceanu L, Matlaf L, Bezrookove V, et al. Human Cytomegalovirus US28 Found in Glioblastoma Promotes an Invasive and Angiogenic Phenotype. Cancer Res. 2011; 71:6643-6653. [PubMed: 21900396]

12. Mitchell D, Archer GE, Bigner DD, et al. Efficacy of a phase II vaccine targeting Cytomegalovirus antigens in newly diagnosed. GBM. 2008:2042.

13. Bollard CM, Aguilar L, Straathof KC, et al. Cytotoxic T Lymphocyte Therapy for Epstein-Barr Virus+ Hodgkin's Disease. J Exp Med. 2004; 200:1623-1633. [PubMed: 15611290]

14. Heslop HE, Slobod KS, Pule MA, et al. Long-term outcome of EBV-specific T-cell infusions to prevent or treat EBV-related lymphoproliferative disease in transplant recipients. Blood. 2010; 115:925-935. [PubMed: 19880495]

15. Leen AM, Rooney CM, Foster AE. Improving T cell therapy for cancer. Annu Rev Immunol. 2007; 25:243-265. [PubMed: 17129181]

16. Lucas KG, Salzman D, Garcia A, et al. Adoptive immunotherapy with allogeneic Epstein-Barr virus (EBV)-specific cytotoxic T-lymphocytes for recurrent, EBV-positive Hodgkin disease. Cancer. 2004; 100:1892-1901. [PubMed: 15112270]

17. Leen AM, Myers GD, Sili U, et al. Monoculture-derived T lymphocytes specific for multiple viruses expand and produce clinically relevant effects in immunocompromised individuals. Nat Med. 2006; 12:1160-1166. [PubMed: 16998485]

18. Bollard CM, Straathof K, Huls MH, et al. Cytotoxic T Lymphocyte Therapy for EBV positive Hodgkin's disease. Blood. 2003; 102:901a.

19. Gottschalk S, Heslop H, Rooney C. Adoptive Immunotherapy for EBV-associated Malignancies. Leuk Lymphoma. 2005; 46:1-10. [PubMed: 15621775]

20. Louis CU, Straathof K, Bollard CM, et al. Adoptive transfer of EBV-specific T cells results in sustained clinical responses in patients with locoregional nasopharyngeal carcinoma. $\mathrm{J}$ Immunother. 2010; 33:983-990. [PubMed: 20948438]

21. Scheurer ME, Bondy ML, Aldape KD, et al. Detection of human cytomegalovirus in different histological types of gliomas. Acta Neuropathol. 2008; 116:79-86. [PubMed: 18351367] 
22. Ahmed N, Salsman VS, Kew Y, et al. HER2-specific T cells target primary glioblastoma stem cells and induce regression of autologous experimental tumors. Clin Cancer Res. 2010; 16:474485. [PubMed: 20068073]

23. Fujita Y, Leen AM, Sun J, et al. Exploiting cytokine secretion to rapidly produce multivirusspecific T cells for adoptive immunotherapy. J Immunother. 2008; 31:665-674. [PubMed: 18600178]

24. Hanley PJ, Shaffer DR, Cruz CR, et al. Expansion of T cells targeting multiple antigens of cytomegalovirus, Epstein-Barr virus and adenovirus to provide broad antiviral specificity after stem cell transplantation. Cytotherapy. 2011

25. Mitchell DA, Xie W, Schmittling R, et al. Sensitive detection of human cytomegalovirus in tumors and peripheral blood of patients diagnosed with glioblastoma. Neuro Oncol. 2008; 10:10-18. [PubMed: 17951512]

26. Papagno L, Almeida JR, Nemes E, et al. Cell permeabilization for the assessment of T lymphocyte polyfunctional capacity. J Immunol Methods. 2007; 328:182-188. [PubMed: 17920073]

27. Harari A, Enders FB, Cellerai C, et al. Distinct profiles of cytotoxic granules in memory CD8 T cells correlate with function, differentiation stage, and antigen exposure. J Virol. 2009; 83:28622871. [PubMed: 19176626]

28. Cheung AK, Abendroth A, Cunningham AL, et al. Viral gene expression during the establishment of human cytomegalovirus latent infection in myeloid progenitor cells. Blood. 2006; 108:36913699. [PubMed: 16931631]

29. Dziurzynski K, Wei J, Qiao W, et al. Glioma-associated cytomegalovirus mediates subversion of the monocyte lineage to a tumor propagating phenotype. Clin Cancer Res. 2011; 17:4642-4649. [PubMed: 21490182]

30. Waziri A. Glioblastoma-derived mechanisms of systemic immunosuppression. Neurosurg Clin N Am. 2010; 21:31-42. [PubMed: 19944964]

31. Avril T, Vauleon E, Tanguy-Royer S, et al. Mechanisms of immunomodulation in human glioblastoma. Immunotherapy. 2011; 3:42-44. [PubMed: 21524170]

32. Gustafson MP, Lin Y, New KC, et al. Systemic immune suppression in glioblastoma: the interplay between CD14+HLA-DRlo/neg monocytes, tumor factors, and dexamethasone. Neuro Oncol. 2010

33. Sippel TR, White JT, Nag K, et al. Neutrophil Degranulation and Immunosuppression in Patients with Glioblastoma: Restoration of Cellular Immune Function by Targeting Arginase I. Clin Cancer Res. 2011

34. Gandhi MK, Lambley E, Duraiswamy J, et al. Expression of LAG-3 by tumor-infiltrating lymphocytes is coincident with the suppression of latent membrane antigen-specific CD8+ T-cell function in Hodgkin lymphoma patients. Blood. 2006; 108:2280-2289. [PubMed: 16757686]

35. Gilbert MJ, Riddell SR, Plachter B, et al. Cytomegalovirus selectively blocks antigen processing and presentation of its immediate-early gene product. Nature. 1996; 383:720-722. [PubMed: 8878482]

36. Jones TR, Hanson LK, Sun L, et al. Multiple independent loci within the human cytomegalovirus unique short region down-regulate expression of major histocompatibility complex class I heavy chains. J Virol. 1995; 69:4830-4841. [PubMed: 7609050]

37. Horn B, Bao L, Dunham K, et al. Infusion of cytomegalovirus specific cytotoxic T lymphocytes from a sero-negative donor can facilitate resolution of infection and immune reconstitution. Pediatr Infect Dis J. 2009; 28:65-67. [PubMed: 19034064]

38. Bunde T, Kirchner A, Hoffmeister B, et al. Protection from cytomegalovirus after transplantation is correlated with immediate early 1-specific CD8 T cells. J Exp Med. 2005; 201:1031-1036. [PubMed: 15795239]

39. Liao X, Li Y, Bonini C, et al. Transfection of RNA encoding tumor antigens following maturation of dendritic cells leads to prolonged presentation of antigen and the generation of high-affinity tumor-reactive cytotoxic T lymphocytes. Mol Ther. 2004; 9:757-764. [PubMed: 15120337]

40. Bollard CM, Gottschalk S, Leen AM, et al. Complete responses of relapsed lymphoma following genetic modification of tumor-antigen presenting cells and T-lymphocyte transfer. Blood. 2007; 110:2838-2845. [PubMed: 17609424] 
41. Heslop HE, Slobod KS, Pule MA, et al. Long-term outcome of EBV-specific T-cell infusions to prevent or treat EBV-related lymphoproliferative disease in transplant recipients. Blood. 2010; 115:925-935. [PubMed: 19880495]

42. Prins RM, Cloughesy TF, Liau LM. Cytomegalovirus immunity after vaccination with autologous glioblastoma lysate. N Engl J Med. 2008; 359:539-541. [PubMed: 18669440]

43. Prins RM, Soto H, Konkankit V, et al. Gene expression profile correlates with T-cell infiltration and relative survival in glioblastoma patients vaccinated with dendritic cell immunotherapy. Clin Cancer Res. 2011; 17:1603-1615. [PubMed: 21135147] 
A

CMV.pp65

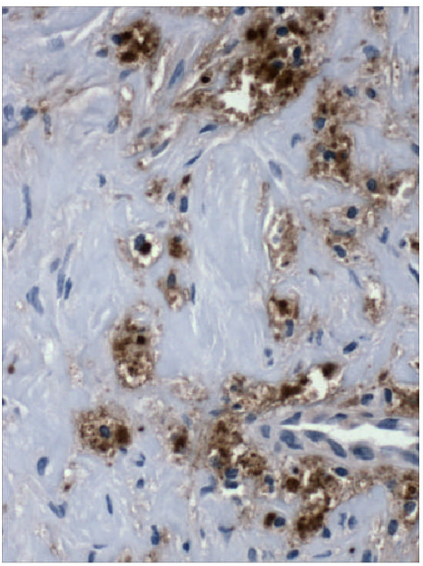

CMV.IE1-72

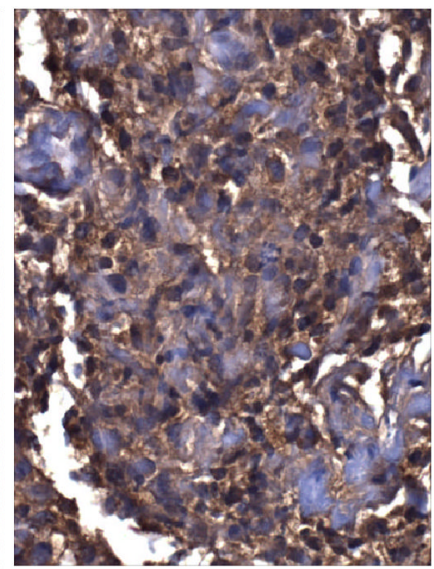

(+)

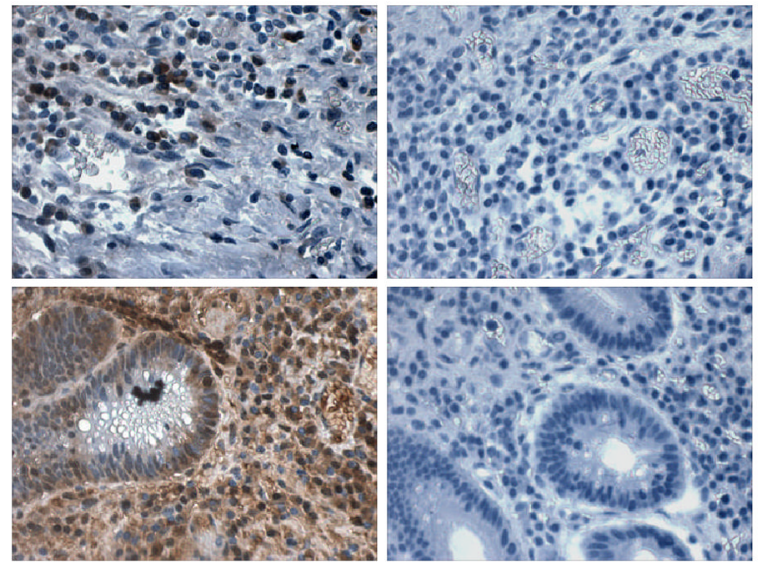

$(-)$

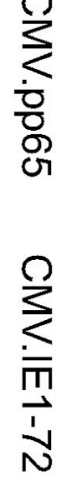

B

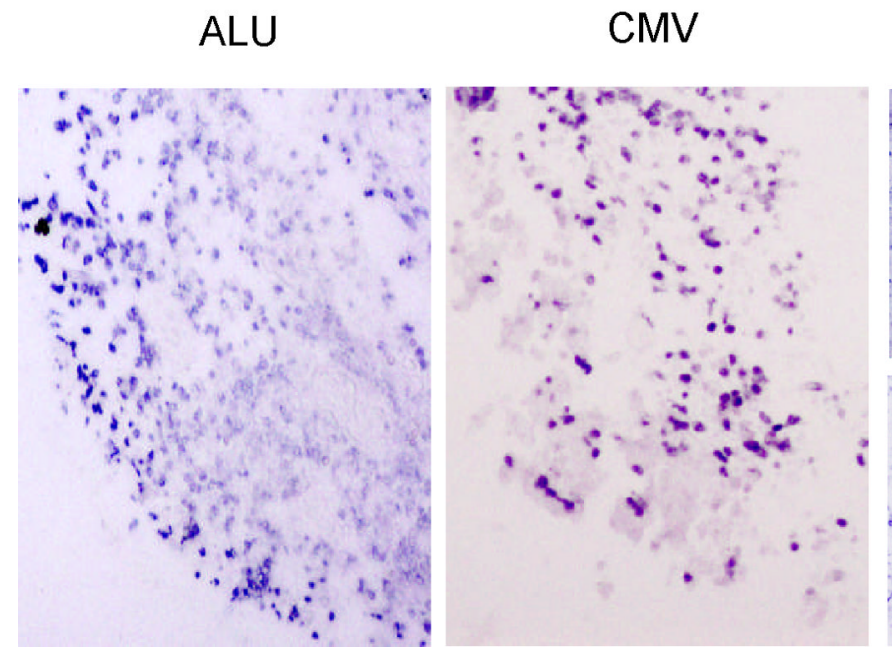

(+)
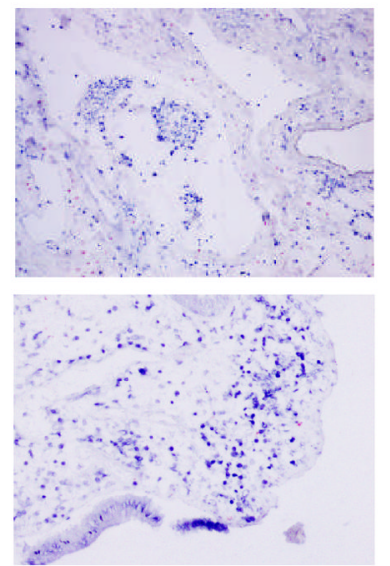

$(-)$

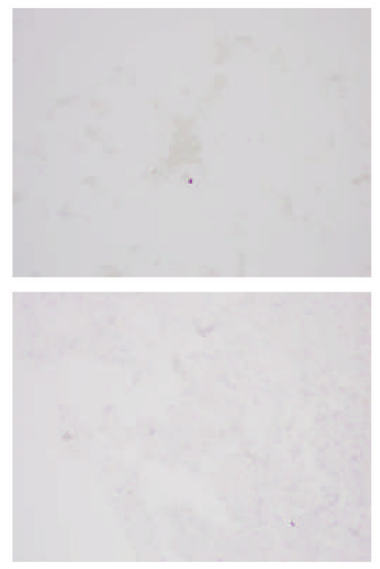

Figure 1. Immunohistochemistry (IHC) for pp65 and IE1 and CMV genome-specific in situ hybridization (ISH) in primary GBM samples

A. IHC was performed on 11 parraffin embedded primary GBM samples. Overall, 5 of 11 samples showed positivity for pp65 while 10 out of 11 patient samples showed positivity for IE1 by IHC. B. Eight of 9 samples were positive using ISH. For both tests, CMV-infected lung tissue was used as a positive control. Elimination of the primary antibody and an ALu tandem probe were used as negative controls for IHC and ISH, respectively. 


\section{CMV.pp65}

A

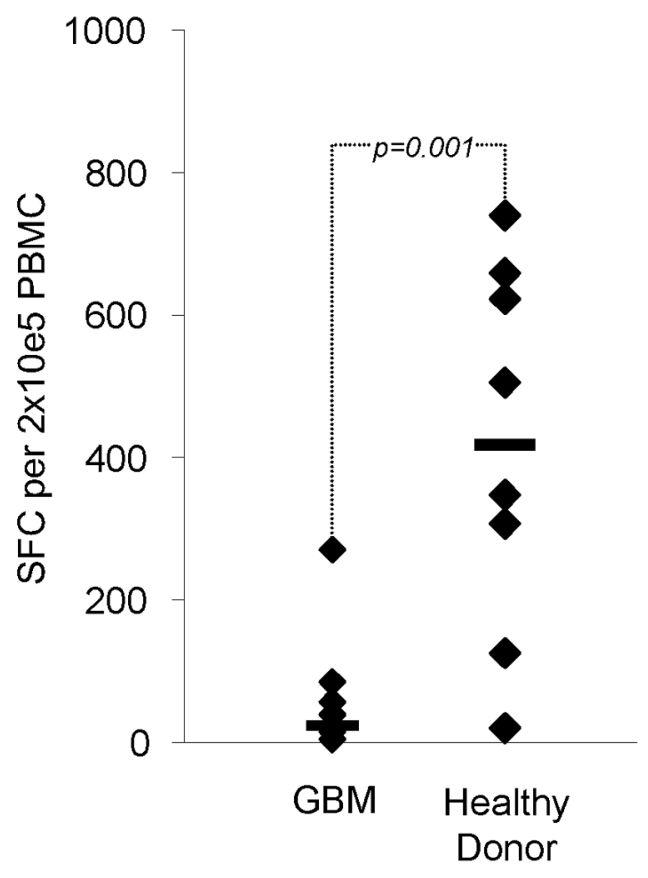

B

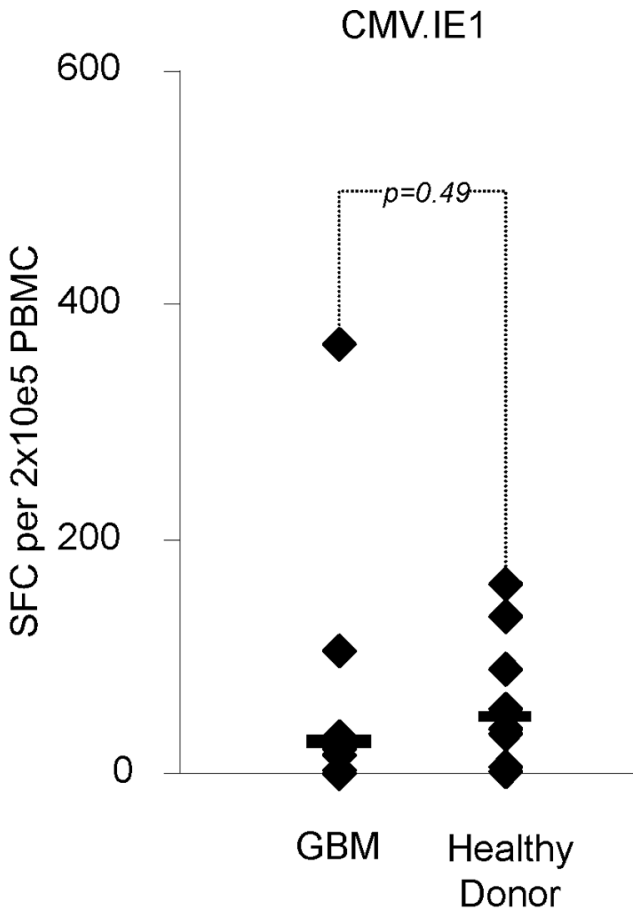

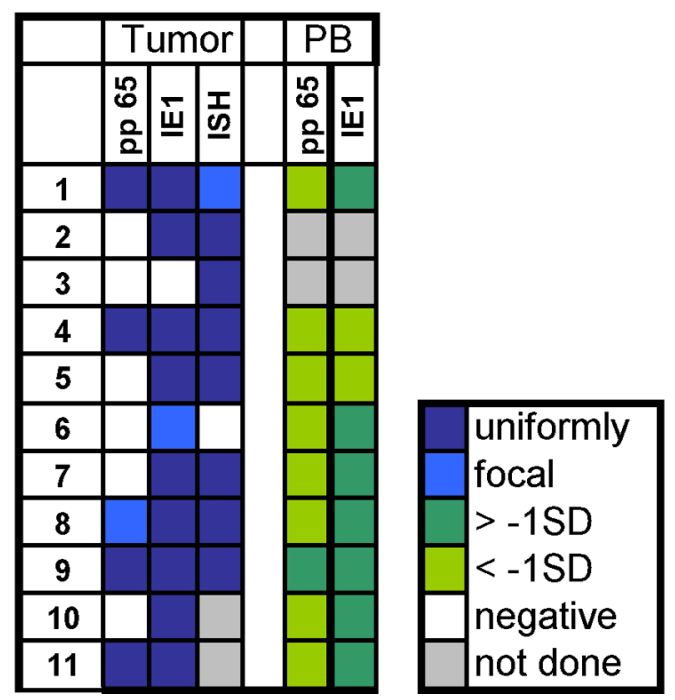

Figure 2. Cellular immune response against CMV in CMV-seropositive GBM patients A. Precursor frequency of pp65- and IE1-specific T cells in the peripheral blood of CMVseropositive GBM patients at diagnosis compared to 7 healthy CMV-seropositive controls. B. Heatmap summarizing the results of the tissue reactivity (IHC and ISH) as well as the corresponding pp65 and IE1 precursor frequency in the peripheral blood (PB) of our patient cohort. SD: standard deviation from the mean value for healthy donors. 
A

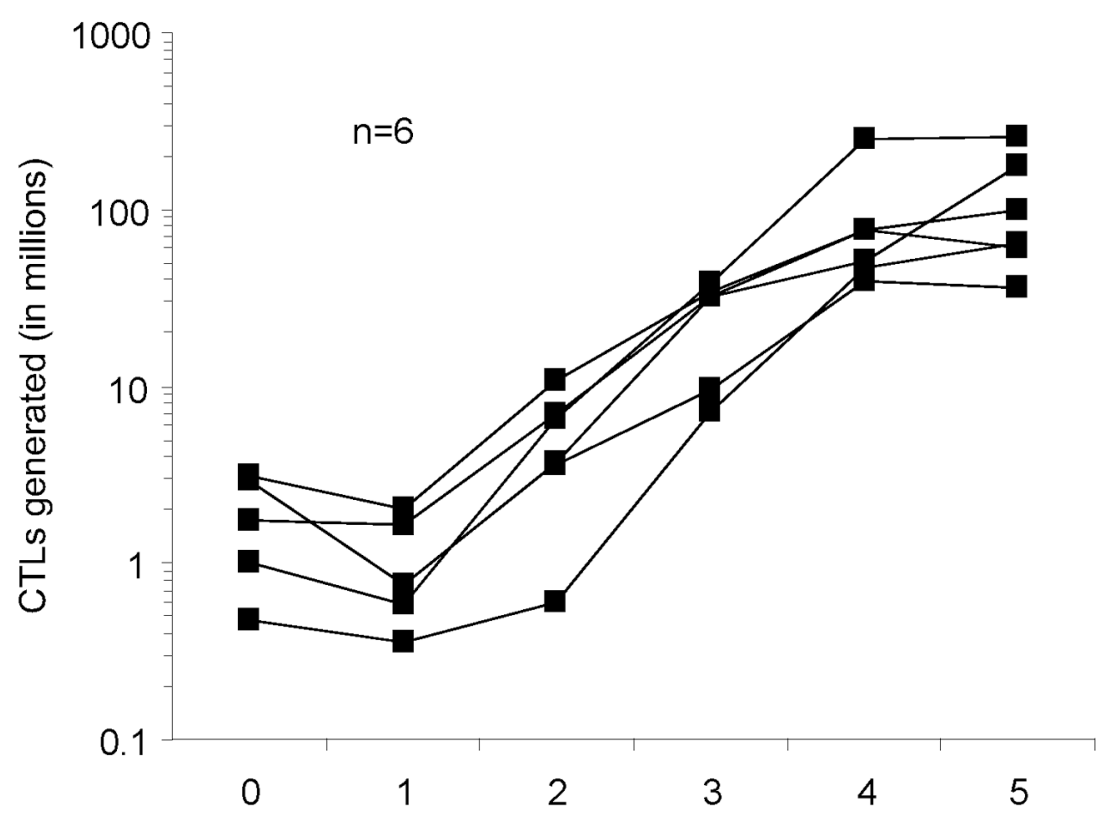

Week

B

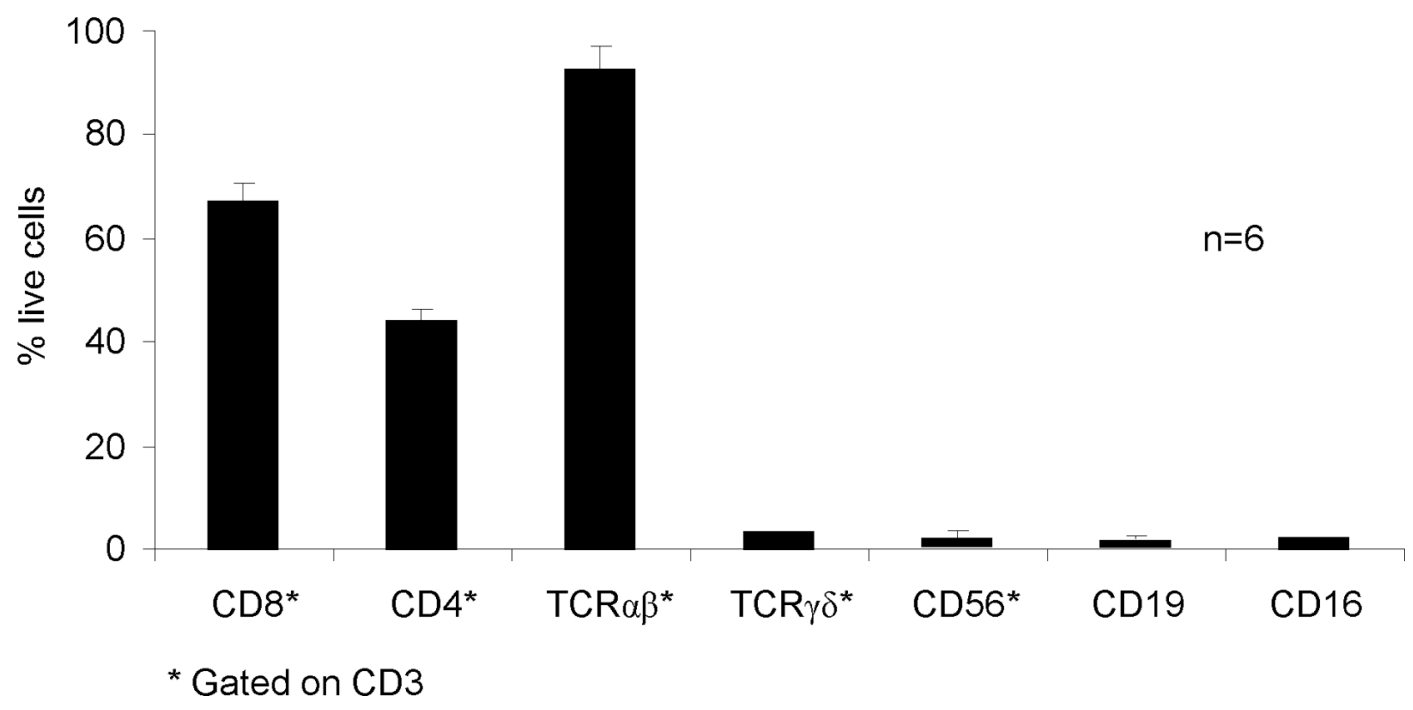

Figure 3. Expansion and phenotype of CMV-specific T-cell lines

A. CMV-specific T-cell lines from 6 patients were expanded to clinically relevant levels within 6 weeks of culture. B. Phenotype of 6 T-cell lines showing predominance of CD8positive TCR $\alpha \beta$ T cells. 
A

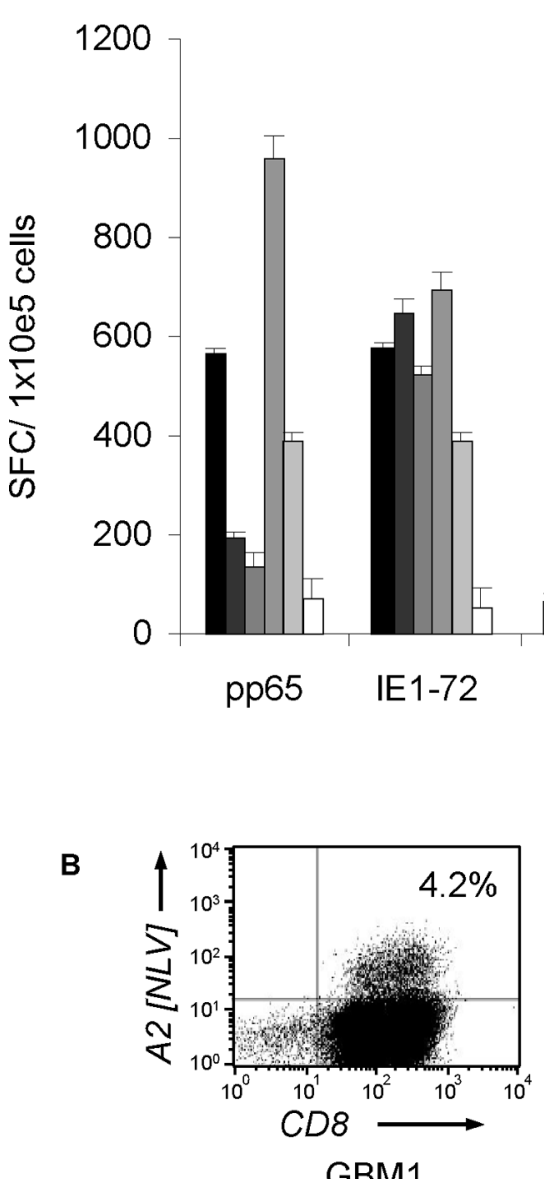

GBM1

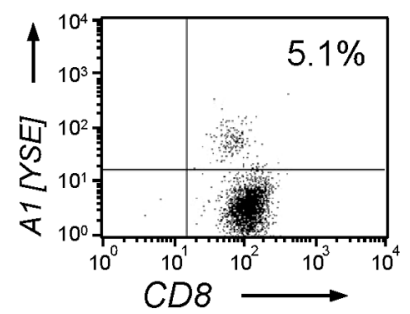

GBM9
- GBM1

- GBM7

$\square$ GBM8

$\square$ GBM9

$\square$ GBM10

$\square$ GBM11

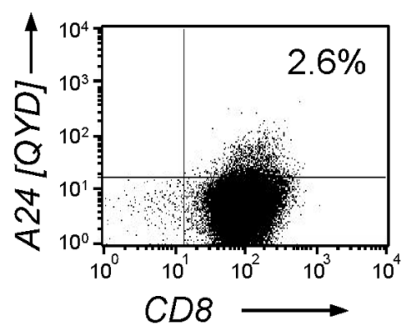

GBM7

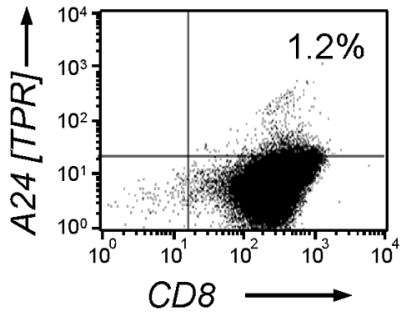

GBM10

* Gated on CD3 and CD8

Figure 4. Functionality of CMV-specific T-cell lines

A. IFN $\gamma$ ELIspot of CMV-specific T cells generated from CMV-seropositive patients. All 6 T-cell lines contained T cells specific for pp65 and IE1. B. Pentamer analysis was performed on 4 representative cell lines confirming the presence of pp65-specific T cells. 

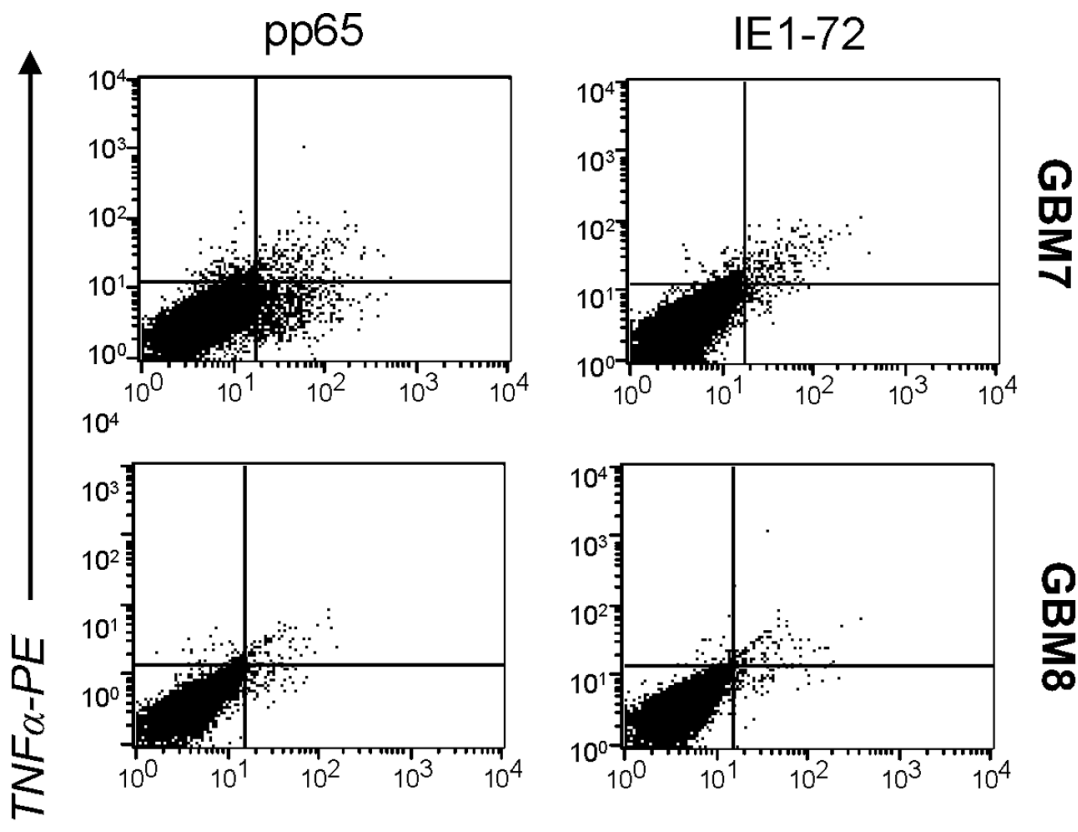

$\underset{\infty}{\infty}$

IFNy-FITC

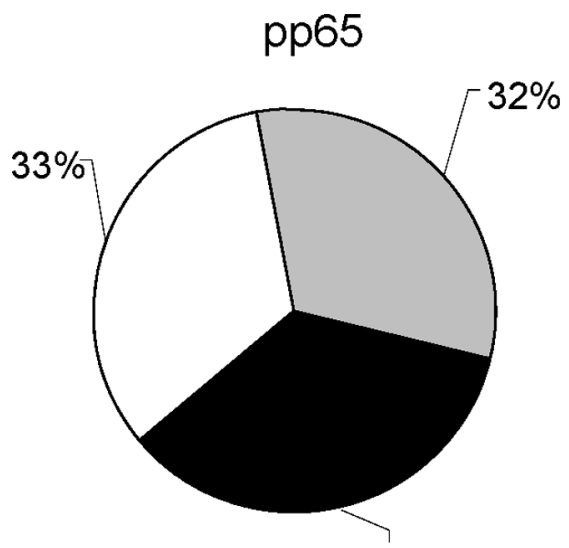

IE1

$35 \%$

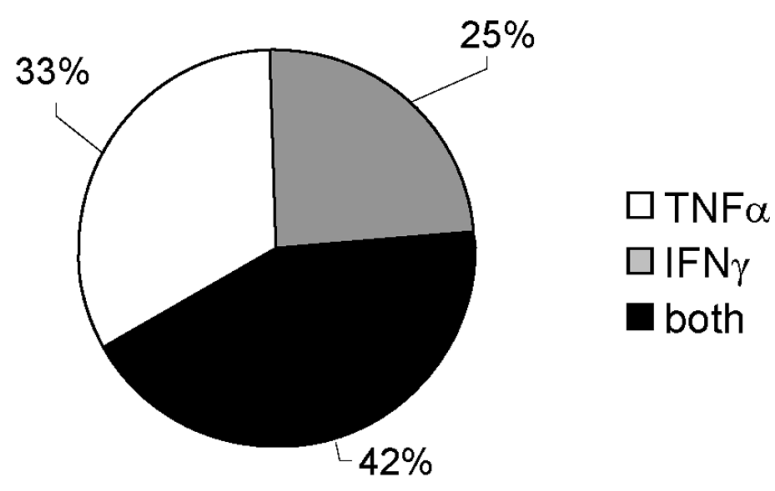

Figure 5. Polyfunctionality of CMV-specific T-cell lines

Upon exposure to pp65 and IE1 pepmixes, 35-42\% of CMV-specific T-cell lines cosecreted IFN $\gamma$ and TNF $\alpha$ indicating polyfunctionality. A representative dot plot is shown for the intracellular cytokine analysis using flowcytometry of CMV-specific CTL line from patient GBM7 and GBM8 and the pie chart summarizes the mean values derived from these two lines. Note that a subset of responding T cells co-secretes both cytokines. 
A.

GBM \#1

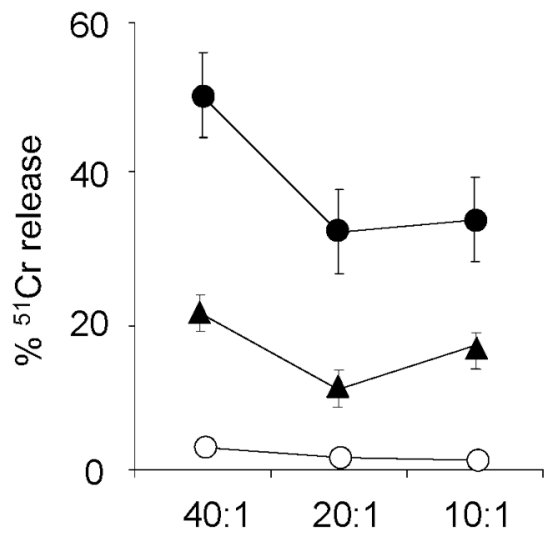

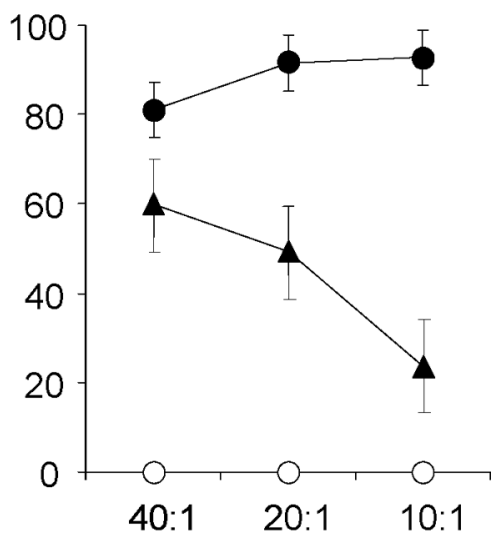

T cell : target ratio

- CMV CTL x pp65 peptide-pulsed autologous GBM

- CMV CTL x IE1-72 peptide-pulsed autologous GBM

-O- CMV CTL $x$ autologous GBM

B.
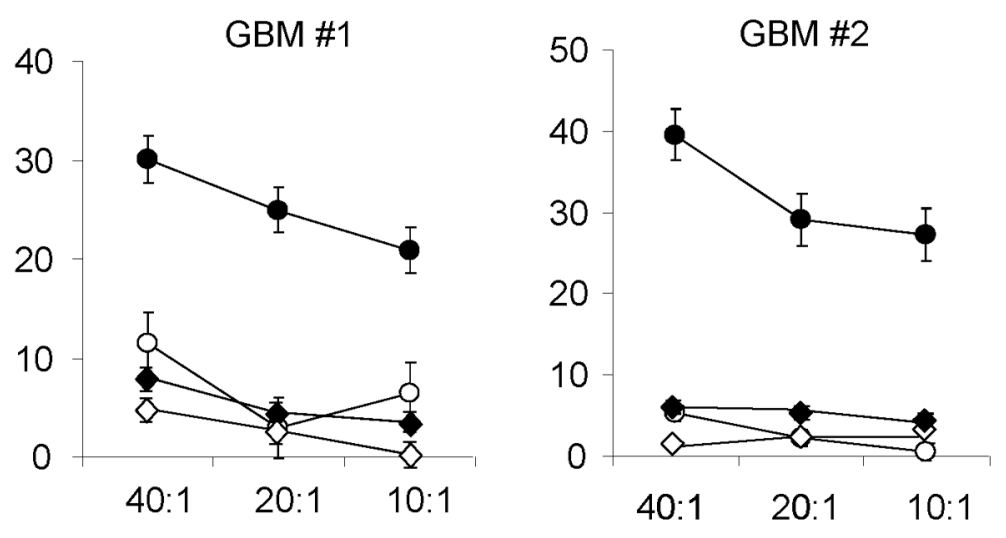

T cell : target ratio

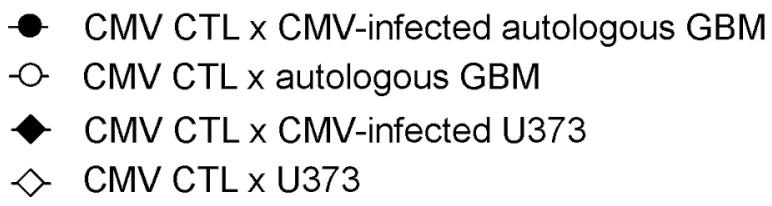

Figure 6. CMV-specific T-cell lines recognize and kill CMV-expressing autologous GBM cells ${ }^{51}$ Chromium-release assay of representative CMV-specific CTLs against autologous (A.) pp65 and IE1 peptides pulsed and (B.) autologous CMV-infected GBMs cells. CMVspecific T-cell lines from representative patients (GBM1: HLA-A29,74, -B18,44; GBM2: HLA-A31,68, -B27,52) recognized and killed CMV-infected as well as pp65- and IE1pulsed autologous glioma cells vs. control. No killing of the HLA-mismatched GBM cell line U373 (HLA-A2, -B18) after infection with CMV VR1814 was observed. 